\title{
CPSARST: an efficient circular permutation search tool applied to the detection of novel protein structural relationships Wei-Cheng Lo and Ping-Chiang Lyu
}

Address: Institute of Bioinformatics and Structural Biology, National Tsing Hua University, Hsinchu 30013, Taiwan.

Correspondence: Ping-Chiang Lyu. Email: pclyu@life.nthu.edu.tw

Published: 18 January 2008

Genome Biology 2008, 9:RII (doi:I0.I I86/gb-2008-9-I-rII)

The electronic version of this article is the complete one and can be found online at http://genomebiology.com/2008/9/I/RII
Received: II September 2007

Revised: 19 November 2007

Accepted: 18 January 2008

() 2008 Lo et al.; licensee BioMed Central Ltd.

This is an open access article distributed under the terms of the Creative Commons Attribution License (http://creativecommons.org/licenses/by/2.0), which permits unrestricted use, distribution, and reproduction in any medium, provided the original work is properly cited.

\begin{abstract}
Circular permutation of a protein can be visualized as if the original amino- and carboxyl termini were linked and new ones created elsewhere. It has been well-documented that circular permutants usually retain native structures and biological functions. Here we report CPSARST (Circular Permutation Search Aided by Ramachandran Sequential Transformation) to be an efficient database search tool. In this post-genomics era, when the amount of protein structural data is increasing exponentially, it provides a new way to rapidly detect novel relationships among proteins.
\end{abstract}

\section{Background}

Circular permutation (CP) in a protein structure is the rearrangement of the amino acid sequence such that the aminoand carboxy-terminal regions are interchanged [1,2]. It can be visualized as if the original termini of the polypeptide were linked and new ones created elsewhere [3,4]. Since the first observation of naturally occurring circular permutations in plant lectins [5], a substantial number of natural examples have been reported, including some bacterial $\beta$-glucanases, swaposins, glucosyltransferases, $\beta$-glucosidases, SLH domains, transaldolases, $\mathrm{C} 2$ domains (for a review, see [6]), FMN-binding proteins [7], double- $\phi \beta$-barrels [8], glutathione synthetases [9], DNA and other methyltransferases $[1,10]$, ferredoxins [11], and proteinase inhibitors [12,13]. In most of the cases, circular permutants (CPs) have conserved function or enzymatic activity [6,14], sometimes with increased functional diversity [15-17].

To reveal the influences of $\mathrm{CP}$ on the structure, function and folding mechanism of proteins, many artificial CPs have been generated, inclusive of trypsin inhibitor, anthranilate isomer- ase, dihydrofolate reductase, $\mathrm{T}_{4}$ lysozyme, ribonucleases, aspartate transcarbamoylase, the $\alpha$-spectrin $\mathrm{SH}_{3}$ domain, the Escherichia coli DsbA protein, ribosomal protein S6 and Bacillus $\beta$-glucanase [18,19]. The outcomes have indicated that three-dimensional structure seems remarkably insensitive to CP [6] and CPs generally retain their biological functions [3,4], although the structural stabilities, the folding nuclei, transition states or pathways might be altered $[18,20,21]$. Since CP generally preserves protein structure and function, with sometimes increased stability or activity, it has been applied to trigger crystallization [22], improve enzyme activities [15], determine critical elements [23,24], and create novel fusion proteins, the tethered sites of which are not confined to the native termini [25-28], such as the famous fluorescent calcium sensor [28].

In spite of these interesting properties and applications, there is still much uncertainty about the genetic mechanisms, the evolutionary importance and the natural prevalence of $\mathrm{CP}$ $[6,18,29,30]$. CPs can arise from posttranslational modifications [5,31] but a majority may arise from genetic events [29]. 
There have been several genetic and evolutionary mechanisms proposed, for instance, duplication/deletion models [6,32], duplication-by-permutation models [1,33], fusion/fission models [2,30], and plasmid-mediated 'cut and paste' [10]. However, which plays the major role or what proportion each mechanism contributes to the evolution of CPs and protein families remains uncertain. Besides, because of the disagreement between definitions of CPs, conflicting conclusions can be observed. In general, previous studies that considered the whole protein as the unit that undergoes CP concluded that $\mathrm{CP}$ is rare in nature $[6,14,30]$ while those viewing the domain as the unit that undergoes CP suggested CP to be frequent $[1,29,34]$.

In this post-genomic era, the amount of protein structure data is increasing exponentially, and plenty of information should be extractable to reveal the natural prevalence and evolutionary mechanism of CP; however, CP search tools are still very rare. It has been indicated that traditional sequence comparison methods are linearly sequential in nature and inefficient at identifying CP [6,35]. Three-dimensional structural comparisons may identify more evolutionarily farrelated CPs [6]; nevertheless, conventional methods such as DALI [36] and CE [37] are also inefficient due to their sequential nature [34]. To detect $\mathrm{CP}$, the most exact approach is to use an algorithm that generates all possible CPs of one protein and subsequently aligns them with another protein to find an alignment better than the linear alignment $[2,38]$, although this is apparently very time-consuming. A few brilliant approaches have been developed to achieve higher efficiency. Uliel et al. [30,38] proposed a heuristic method based on duplicating one of the two protein sequences followed by manual verifications. Though being much faster, it still takes several CPU months to survey tens of thousands of sequences. The requirement of manual examinations also makes it unrealistic for searching large datasets [2]. Weiner et al. [2] condensed amino acid sequences into tiny domain strings to achieve an extremely high speed, scanning hundreds of thousands of sequences in hours; however, without suitable domain annotations or when a CP disrupts a domain, false negatives occur. Structural alignment methods applicable to the identification of CPs have also been developed. For instance, Jung and Lee [29] developed SHEBA to screen the SCOP database. They suggested that CPs are very frequent and many have symmetric structures. However, since internal symmetry may introduce noise into the detection of CPs [39], certain false positive predictions can be produced. Regardless of the capability of detecting distantly related CPs, a pair-wise comparison by structure-based CP-detecting algorithms may take from seconds to minutes [34], making routine database searches infeasible.

\section{Overview of CPSARST}

Here we present CPSARST (Circular Permutation Search Aided by Ramachandran Sequential Transformation), an efficient tool for searching for CPs. It describes three-dimen- sional protein structures as one-dimensional text strings by using a Ramachandran sequential transformation (RST) algorithm [40], which transforms protein structures through a Ramachandran (RM) map organized by nearest-neighbor clustering. This linear encoding methodology converts complicated and time-consuming structural comparison problems into string comparisons that can be done very rapidly. CPSARST has also achieved high efficiency by duplicating the query structure and working through a 'double filter-andrefine' strategy. These approaches are illustrated in Figure 1. A web service and a stand-alone Java program of CPSARST are available at [41]. CPSARST not only inherits the speed advantages of sequence-based methods but retains sensitivity to detect distantly related CPs mostly detectable only by structure-based methods. To the best of our knowledge, it is the first structural similarity search method that makes large scale all-against-all database searches for CP achievable and practicable. We suppose that this procedure can be applied to reveal the evolutionary importance of CP and detect novel protein structural relationships. Several novel CP relationships have been detected by CPSARST and are reported in this article; also, some rational estimations of the prevalence of $\mathrm{CP}$ in protein structural databases have been made by doing all-against-all database searches of non-redundant Protein Data Bank (PDB) and SCOP.

\section{Results \\ Performance on random circular permutants}

Although CPSARST basically uses structurally meaningful RM strings to search protein databases, its algorithm is actually applicable to amino acid sequences. To evaluate their amino acid sequence-based algorithm, Uliel et al. performed in silico random $\mathrm{CP}$ followed by various levels of regular mutations (substitutions, insertions and deletions) on a number of proteins [38]. We adapted this approach in a more thorough manner and developed a random CP dataset containing 20,000 chains (RCP dataset; see Materials and methods) to assess the performance of CPSARST with amino acid sequences. Two parameters were monitored: the proportion of cases in which the exact permutation site was retrieved; and the percentage distance of the retrieved permutation site to the exact one, which is defined as:

$D(\%)=\frac{\text { Number of residues off the exact permutation site }}{\text { Sequence length }} \times 100$

As shown in Figure 2a, the percentage of exact matched cases retrieved by CPSARST remains over $80 \%$ until the sequence identities fall between $40 \%$ and $30 \%$. When we made a $50 \%$ exact matches cut, the results indicated CPSARST ensures that at least $50 \%$ of the retrieved cases are exact as long as the sequence identities are higher than $22 \%$. 


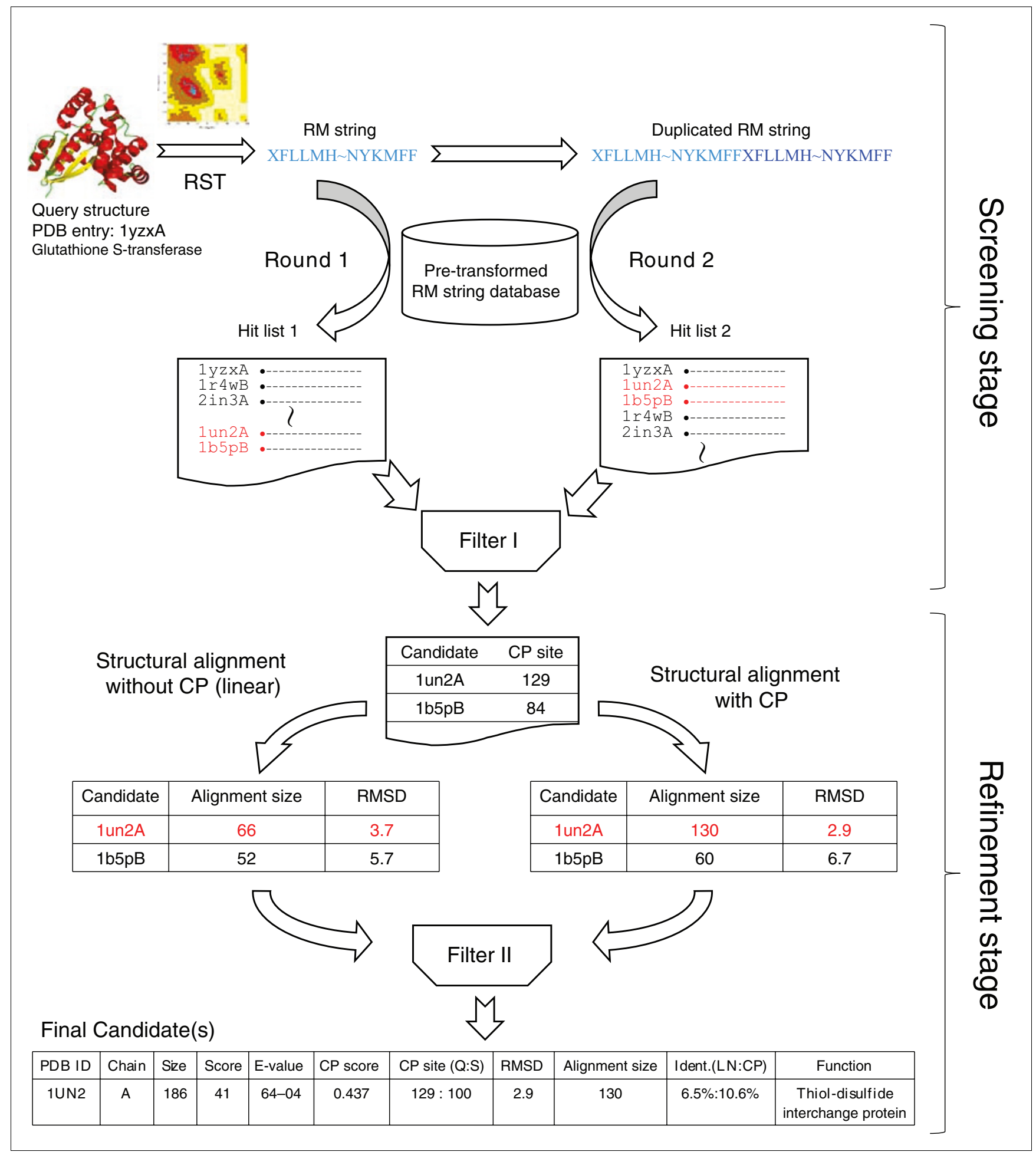

\section{Figure I}

Flowchart of CPSARST. CPSARST uses a 'double filter-and-refine' strategy combining a fast screening and an accurate refinement step, each having two different rounds. In the screening stage, the three-dimensional structure of the query protein is transformed into a one-dimensional structural string by a RST algorithm [40]. This query string is subjected to two rounds of database searches. In round I, it is searched against a pre-transformed structural string database by a heuristic method. In round 2 , it is duplicated prior to the database search. Results of the two rounds are filtered; hits with meaningfully improved similarity scores are considered as CP candidates (colored red). In the refinement stage, candidates are analyzed by an accurate structural alignment algorithm, FAST [63], with and without CP manipulation, to determine their reliabilities and to retrieve permutation sites more precisely. After filtering out improbable cases, final answers with detailed information are output. The example used in this figure is a real case with simplified hit lists. 
(a)

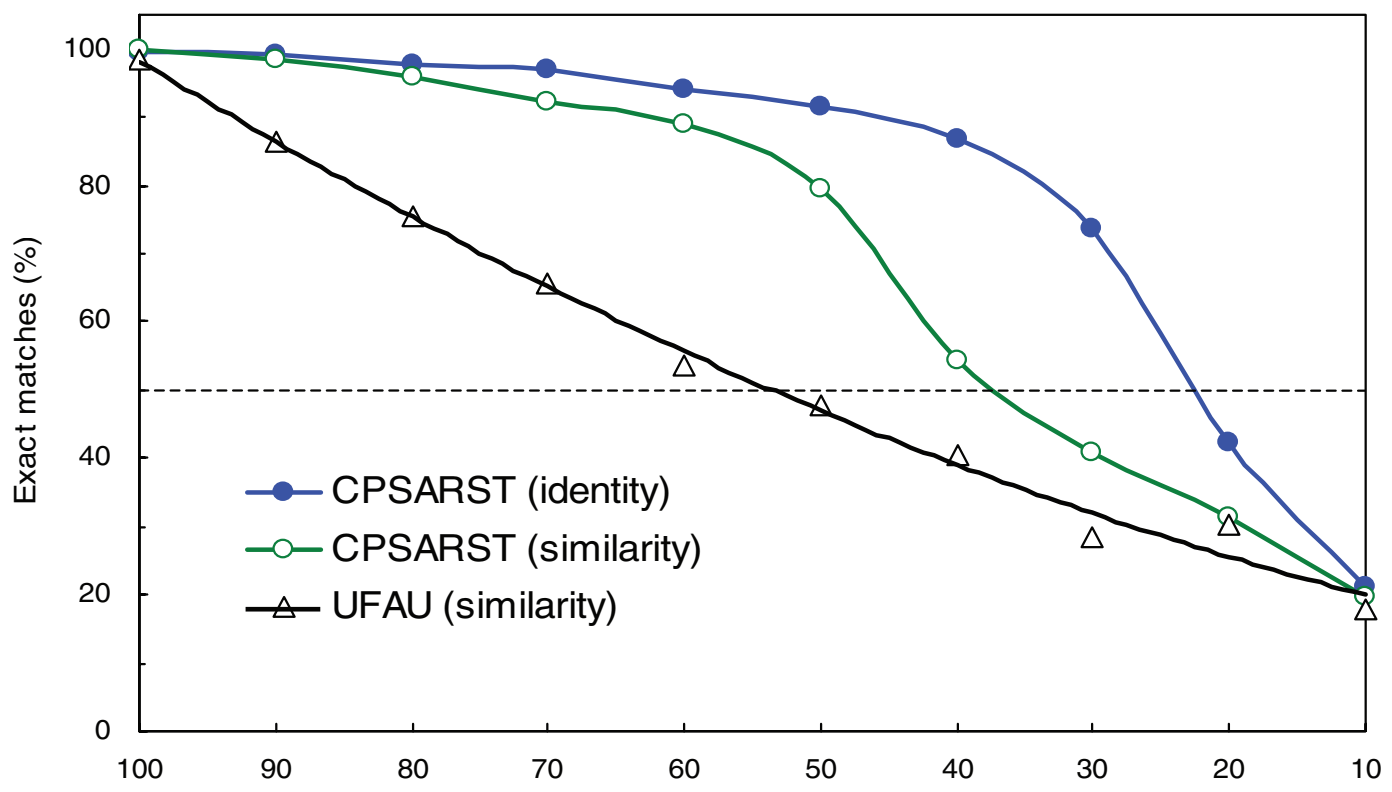

Identity / Similarity (\%)

(b)

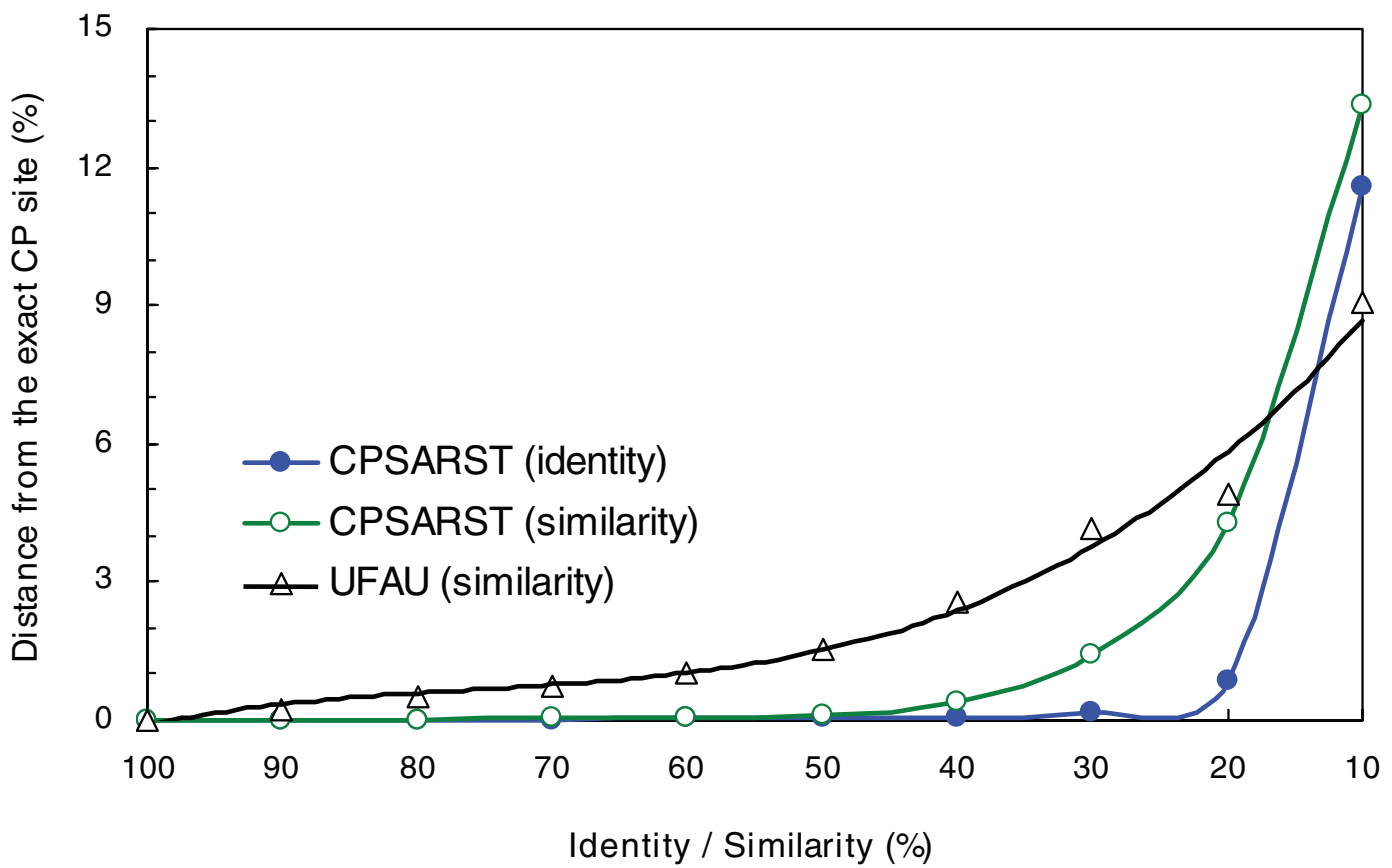

Figure 2

Performance on RCPs. The methodology of CPSARST is not only applicable to structurally meaningful RM strings but also to amino acid sequences. Random CP followed by various degrees of random substitutions, insertions and deletions were performed on 100 amino acid sequences. The performance of CPSARST was monitored by (a) the percentage of cases in which the exact permutation site was retrieved, and (b) the percentage distance of the retrieved permutation site to the exact one. The dashed line in (a) represents a $50 \%$ cut, above which more than half of the permutation sites were exactly predicted. When it only depends on amino acid sequences to detect CP, CPSARST can be reliable even if the identity is as low as $20 \%$. UFAU stands for the CP-detecting method developed by Uliel et al. [38]. 
The curve of the percentage distance of CPSARST has a half hyperbolic shape (Figure 2b). Provided that the sequence identity is $>20 \%$, the percentage distance will be $<1 \%$. Combining these data, we suggest that when our approach is applied to amino acid sequences, it will be reliable in detecting CPs with sequence identities as low as about $20 \%$.

\section{Accuracy evaluations with engineered circular permutants}

Since there are many artificial CPs, each with a definite parent protein, a known permutation site, and sometimes some regular mutations, they provide a good resource to assess the performance of a $\mathrm{CP}$ search method. We used keyword searches to find the engineered CPs recorded in the PDB [42], and subjected them to CPSARST searches. As summarized in Table 1, among the 15 non-redundant cases, all the parent proteins were successfully retrieved. Their average percentage distance is only $0.08 \%$, which means that the CP sites identified are very close to the exact ones, demonstrating the high accuracy of CPSARST for engineered CPs.

\section{Pair-wise comparisons of naturally occurring circular permutants}

To our knowledge, current CP-detecting methods based on structural comparisons work in only a pair-wise fashion. Although CPSARST is a database search procedure, it can be simplified to perform pair-wise comparisons (see Materials and methods). Here, we used naturally occurring CP candidates to test the performance of CPSARST. These candidate pairs were detected by doing all-against-all searches against a non-redundant PDB dataset (see below for details) and then filtering out engineered permutants. The 'structural diversity' defined by Lu [43] that integrates the concepts of normalized alignment size and root mean square distance (RMSD) was used to evaluate the quality of pair-wise comparisons:

$$
\text { structure diversity }=\frac{\text { RMSD }}{\left(\frac{\text { alignment size }}{\operatorname{avg}\left(\mathrm{N}_{\mathrm{q}}, \mathrm{N}_{\mathrm{S}}\right)}\right)^{1.5}}
$$

where $\operatorname{avg}\left(\mathrm{N}_{\mathrm{q}}, \mathrm{N}_{\mathrm{s}}\right)$ is the average size of the query and subject protein. Lower structural diversities stand for higher structural alignment qualities of the assessed methods. The results are listed in Tables 2 and 3. In terms of structural diversity, the performance of CPSARST is better than that of SHEBA [11] and is comparable to SAMO [34]. In addition, CPSARST is 9.3 times faster than SAMO in these pair-wise comparisons (Table 2). Protein size has no effect on the alignment qualities of these structure-based methods while the running time increases as the size becomes larger. This increase in running time is lowest for CPSARST, apparently much lower than that of SAMO. Sequence identities greatly influence the performance, especially for SHEBA (Table 3). The differences in structural diversities calculated by CPSARST and SAMO are not obvious until the sequence identity of the CP pair becomes lower than $20 \%$.

CPSARST runs very rapidly in pair-wise comparisons. When searching databases, its speed will be even higher since it does not work in a pair-wise manner but with a 'double filter-andrefine' strategy. Chen had estimated that using SAMO to

Table I

Retrieved parent proteins of engineered CPs by CPSARST

\begin{tabular}{|c|c|c|c|c|c|c|}
\hline PDB entry & Chain & Size & Function & $\begin{array}{l}\text { Parent structure/ } \\
\text { recorded CP site }\end{array}$ & $\begin{array}{l}\text { Retrieved structure/ } \\
\text { determined } \mathrm{CP} \text { site }\end{array}$ & $D(\%)^{*}$ \\
\hline$\underline{\mathrm{IAJK}}$ & $A, B$ & 214 & Circularly permuted (I-3,I-4)-beta-D-glucan 4-glucanohydrolase H & $2 \mathrm{AYH} / 84$ & $2 \mathrm{AYH} / 84$ & 0.00 \\
\hline$\underline{\mathrm{IAJO}}$ & $A, B$ & 214 & Circularly permuted (I-3,I-4)-beta-D-glucan 4-glucanohydrolase H & $2 \mathrm{AYH} / \mathrm{I} 27$ & $2 \mathrm{AYH} / \mathrm{I} 27$ & 0.00 \\
\hline$\underline{\mathrm{ALQ}}$ & & 266 & CP254 beta-lactamase & $3 B L M / 254$ & $3 B L M / 254$ & 0.00 \\
\hline$\underline{\mathrm{IBD} 7}$ & $A, B$ & 176 & Circularly permuted BB2-crystallin & IBLBC/87 & IBLBC/87 & 0.00 \\
\hline$\underline{I C P M}$ & & 214 & Glucanase & $2 \mathrm{AYH} / 59$ & $2 \mathrm{AYH} / 59$ & 0.00 \\
\hline$\underline{I C P N}$ & & 208 & Glucanase & $2 \mathrm{AYH} / 59$ & $2 \mathrm{AYH} / 59$ & 0.00 \\
\hline$\underline{I F W 8}$ & A & 416 & Phosphoglycerate kinase & $3 P G K / 72$ & 3PGK/73 & 0.24 \\
\hline$\underline{1 G 2 B}$ & A & 62 & Spectrin alpha chain & ISHG/47 & ISHG/47 & 0.00 \\
\hline$\underline{\mathrm{NN} 02}$ & A & 102 & Cyanovirin- $\mathrm{N}$ & $2 \mathrm{EZM} / 50$ & $2 \mathrm{EZM} / 5 \mathrm{I}$ & 0.98 \\
\hline$\underline{\text { IP5C }}$ & A-D & 167 & Lysozyme & ILW9A/I2 & ILW9A/I2 & 0.00 \\
\hline$\underline{\text { ISWF }}$ & A-D & 128 & Circularly permuted core-streptavidin E5I/A46 & ISTP/5 | & ISTP/5 | & 0.00 \\
\hline ISWG & A-D & 128 & Circularly permuted core-streptavidin E5I/A46 & ISTP/5 | & ISTP/5 | & 0.00 \\
\hline$\underline{\text { ITUC }}$ & & 63 & alpha-Spectrin & ISHG/20 & ISHG/20 & 0.00 \\
\hline$\underline{\text { ITUD }}$ & & 62 & alpha-Spectrin & ISHG/48 & ISHG/48 & 0.00 \\
\hline$\underline{\text { IUN2 }}$ & A & 197 & Thiol-disulfide interchange protein & $\mathrm{IA} 2 \mathrm{~J} / 100$ & $\mathrm{IA} 2 \mathrm{~J} / 100$ & 0.00 \\
\hline Average & & & & & & 0.08 \\
\hline
\end{tabular}

*Percentage distance of the retrieved permutation site to the exact one. See text for definition. 
Table 2

\begin{tabular}{|c|c|c|c|c|c|c|c|}
\hline \multirow{2}{*}{$\begin{array}{l}\text { Length of the query } \\
\text { protein (residues) }\end{array}$} & \multirow{2}{*}{$\begin{array}{l}\text { No. of } \\
\text { candidate } \\
\text { CP pairs }\end{array}$} & \multicolumn{2}{|c|}{ CPSARST } & \multicolumn{2}{|c|}{ SHEBA } & \multicolumn{2}{|c|}{ SAMO } \\
\hline & & $\begin{array}{l}\text { Structural } \\
\text { diversity }\end{array}$ & $\begin{array}{l}\text { Average } \\
\text { running time (s) }\end{array}$ & $\begin{array}{c}\text { Structural } \\
\text { diversity }\end{array}$ & $\begin{array}{c}\text { Average } \\
\text { running time (s) }\end{array}$ & $\begin{array}{l}\text { Structural } \\
\text { diversity }\end{array}$ & $\begin{array}{c}\text { Average } \\
\text { running time (s) }\end{array}$ \\
\hline$\leq 100$ & 135 & 5.269 & 0.245 & 6.600 & 0.506 & 4.024 & 0.765 \\
\hline $100-150$ & 223 & 6.629 & 0.381 & 10.255 & 0.767 & 4.359 & 2.243 \\
\hline $150-200$ & 464 & 6.105 & 0.520 & 12.730 & 0.955 & 4.591 & 3.554 \\
\hline $200-250$ & 177 & 4.410 & 0.922 & 10.683 & 1.390 & 3.499 & 6.793 \\
\hline $250-300$ & 39 & 6.645 & 1.063 & 11.092 & 1.774 & 4.277 & 10.820 \\
\hline$>300$ & 30 & 6.918 & 1.894 & 6.976 & 2.224 & 4.423 & 22.345 \\
\hline Average & & & 0.838 & & 1.269 & & 7.753 \\
\hline
\end{tabular}

compare two proteins mostly took around ten seconds [34]. Searching the current PDB (approximately 90,000 polypeptides) by one-against-all comparisons will, therefore, require over 15,000 minutes. However, CPSARST can do this oneagainst-all comparison in 1.7 minutes (see below). As shown by these naturally occurring cases, CPSARST achieves a high speed with a reasonable compromise in alignment accuracy.

\section{Protein structural database searches}

To examine the database searching performance of CPSARST, two non-redundant protein databases were used, the 90\% sequence identity subsets of PDB (January 2007) and the ASTRAL SCOP dataset (v.1.71) [44], which were abbreviated as nrPDB-90 (14,422 polypeptides) and nrSCOP90 (11,688 domains), respectively (see Additional data files 1 and 2 for lists of entry IDs). As summarized in Table 4, the allagainst-all survey of large protein databases like nrPDB-90 took 65.7 hours. Since there were approximately 200 million protein pairs for this database $(14,422 \times 14,422)$, these data demonstrated that CPSARST could scan around 52,800 pairs per minute. At this speed, a full search of the current PDB could be finished in 1.7 minutes per query protein. In comparison with 6.4 minutes required by the sequence-based UFAU method (developed by S Uliel, A Fliess, A Amir and R Unger) [38] and 15,000 minutes by the structure-based SAMO [34], CPSARST runs fairly fast. Besides, CPSARST gives the user two parameters, expectation value (E-value) and CP score, to evaluate the significance of the retrieved information.

As a database search method, CPSARST provides a list of hits ranked by the statistically meaningful E-value. Given that a hit has a similarity score $S$, the E-value is the number of different alignments with scores equivalent to or better than $S$ that are expected to occur in this particular database search by chance [45-47]. A lower E-value indicates a higher significance for the score. This statistical significance is a useful indicator of the reliability of the search results.

To determine the extent to which two proteins are related by a $\mathrm{CP}$, we used the CP scoring scheme described by Vesterstrom and Taylor [39]. The minimum value of this CP score is -1 for a pair of completely linearly aligned proteins, and its maximum value is 1 for a perfect $\mathrm{CP}$ alignment. In general, a small positive CP score indicates that only a small fraction of the protein is permutated while a larger one reveals that the $\mathrm{CP}$ site is closer to the middle of the polypeptide chain.

Table 3

Performance of pair-wise comparisons for natural candidate CP pairs over various sequence identities

\begin{tabular}{lcccc}
\hline \multirow{2}{*}{ Identity (\%) } & No. of candidate CP pairs & & Structural diversity \\
\cline { 3 - 5 } & & CPSARST & SHEBA & SAMO \\
\hline$\leq 10$ & 823 & 6.309 & 11.180 & 4.396 \\
$10-20$ & 152 & 5.864 & 13.881 & 4.994 \\
$20-30$ & 11 & 3.581 & 4.506 & 3.363 \\
$30-40$ & 33 & 1.868 & 3.284 & 2.210 \\
$40-50$ & 40 & 1.755 & 3.096 & 1.544 \\
$>50$ & 9 & 1.385 & 2.247 & 1.520 \\
\hline
\end{tabular}


Table 4

\begin{tabular}{lcc}
\hline Statistics of protein structural database searches & & \\
\hline Database & nrPDB-90 & nrSCOP-90 \\
\hline No. of proteins & 14,422 & 11,688 \\
No. of candidate pairs & & 1,802 \\
$\quad$ Detected by amino acid sequence & 5,020 & 196,533 \\
$\quad$ Detected only by Ramachandran string & 252,287 & 4,228 \\
$\quad$ Confirmed after the refinement stage & 2,911 & 1,161 \\
$\quad$ Total & 682 & $136.6 \times 106$ \\
$\quad$ Symmetric CP & $208.0 \times 106$ & 1,974 \\
Total no. of protein pairs & 3,942 & 69,204 \\
Total running time (minutes) & 52,764 & \\
No. of protein pairs scanned per minute & & \\
\hline
\end{tabular}

In the survey of nrPDB-90 and nrSCOP-90, we had set the RMSD cutoff as $5 \AA$, the E-value cutoff as 0.1 and the CP score threshold as 0.2. Under these criteria, 2,911 and 4,228 candidate pairs were identified in nrPDB-90 and nrSCOP-90, respectively. For nrPDB-90, the 2,911 candidate pairs consisted of 1,822 different polypeptides, that is $12.6 \%$ (1,822 of 14,422) of the polypeptides have $\mathrm{CP}$ relationships with at least one other polypeptide. For nrSCOP-90, the proportion is $17.6 \%$ (2,060 of 11,688$)$.

\section{Novel circular permutation family detected by CPSARST}

After visual inspections of superimposed CP pairs detected by CPSARST, we found that it is possible for proteins with very different functions and divergent amino acid sequences to share CP relationships structurally, forming novel CP families, which are difficult to identify using conventional comparison methods. For instance, although glycine betainebinding proteins (GBBPs), molybdate-binding proteins and Klebsiella aerogenes cysteine regulon transcriptional activator CysB share similar overall structures when judged by the naked eye, their sequence identity is low ( $<24 \%$; calculated by FASTA [48]) and structural relatedness is hard to detect by conventional methods (Figure 3). CPSARST detected CP relationships among GBBPs themselves and among these three groups of proteins. To our knowledge, these CP relationships have not been reported previously. Figure 3 illustrates that the functional and evolutionary relationships among these proteins cannot be correctly determined by their raw sequences; their ligand-interacting residues are not wellaligned and proteins with more similar functions are separated while those with less similar functions cluster together in the phylogram tree. However, the circularly permuted sequences retrieved by CPSARST can be well-aligned and the phylogram tree agrees with the functional relatedness among these proteins. A superimposition of six of these proteins is also shown in Figure 3 to demonstrate their structural similarity and the conserved position of their ligand binding pockets.

\section{Circular permutants detected by CPSARST}

We examined the candidate pairs detected by CPSARST with RMSD $\leq 3.5 \AA$ by visual inspection of superimposed structures and found that approximately 55\%, 25\% and $20 \%$ are mainly alpha, mainly beta, and alpha-beta structures, respectively. These CP pairs are listed, each with a superimposed image, in Additional data file 3; many well-known CP cases are listed, such as some lectins, glucanases, transaldolases, methyltransferases, ferredoxins, protease inhibitors and GTPases. Furthermore, a large number of these CP relationships have not been reported yet, for example, chorismate mutases ([PDB:1 $1 \mathrm{CSM}]$ versus [PDB:2AO2]); some (approximately 20\%) even involve hypothetical proteins, implying that CPSARST can be applied to suggest possible functions for hypothetical proteins.

Rat Rab3A is a small G protein with GTPase activity [49]. CPSARST detected that it has a CP relationship with a conserved hypothetical protein YlqF from Bacillus subtilis, the structure of which was determined by the New York Structural Genomics Research Consortium. When we searched with YlqF against the PDB using the DALI server [50], a number of isomerases, elongation factors, $\mathrm{G}$ proteins, transferases and other hypothetical proteins with inconvincible quality of structural alignments, i.e. small alignment sizes and large RMSD, were returned (Additional data file 4). However, CPSARST detected that many $\mathrm{G}$ proteins superimpose well with YlqF, suggesting that it may possess GTP binding/ GTPase activity (Table 5). Figure 4 shows that DALI can only partially align Rab3A and YlqF (alignment size, 96; RMSD, 2.9 A), while CPSARST successfully detects the CP relationship between them (alignment size, 130; RMSD, $3.2 \AA$ ).

Jung and Lee [29] suggested that when a pair of proteins can be well-aligned, with or without $\mathrm{CP}$ of the sequences, they are symmetric CPs. Considering this definition, proteins containing repeats or duplications will be included. However, Uliel et al. [30] supposed that these should be differentiated from true CPs. In our point of view, the certification of a CP 
(a)

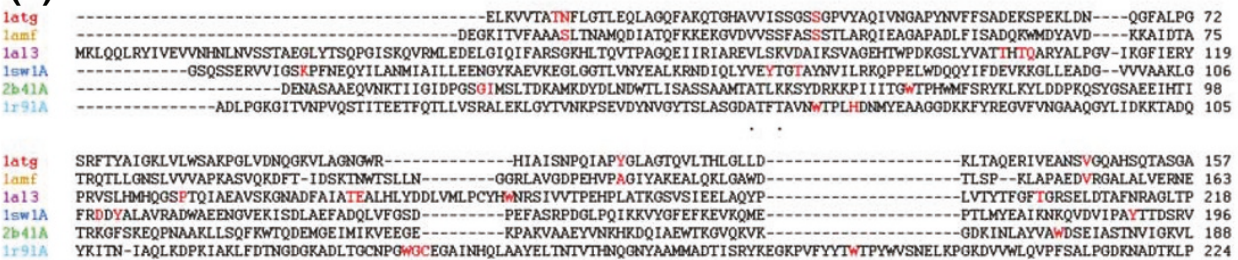
:

ADLGFVALAQIIQAAAKIPGSHWFPPANYYEPIVQQAVITRST -AERANAEQFMSMMK--GPRAVAIIKAAGYVLPQ---20 231 RIVFTATDADVI KTYVRLGLGVGVIASMAVDPVSDPDLVKLDANGIFSHSTTRIGFRRSTFLRSMYYDF IORFAPHLTRDVVDTAVALRSNEDIEAMFRDIRLPER 324

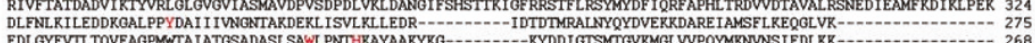

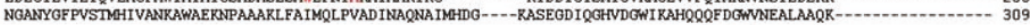
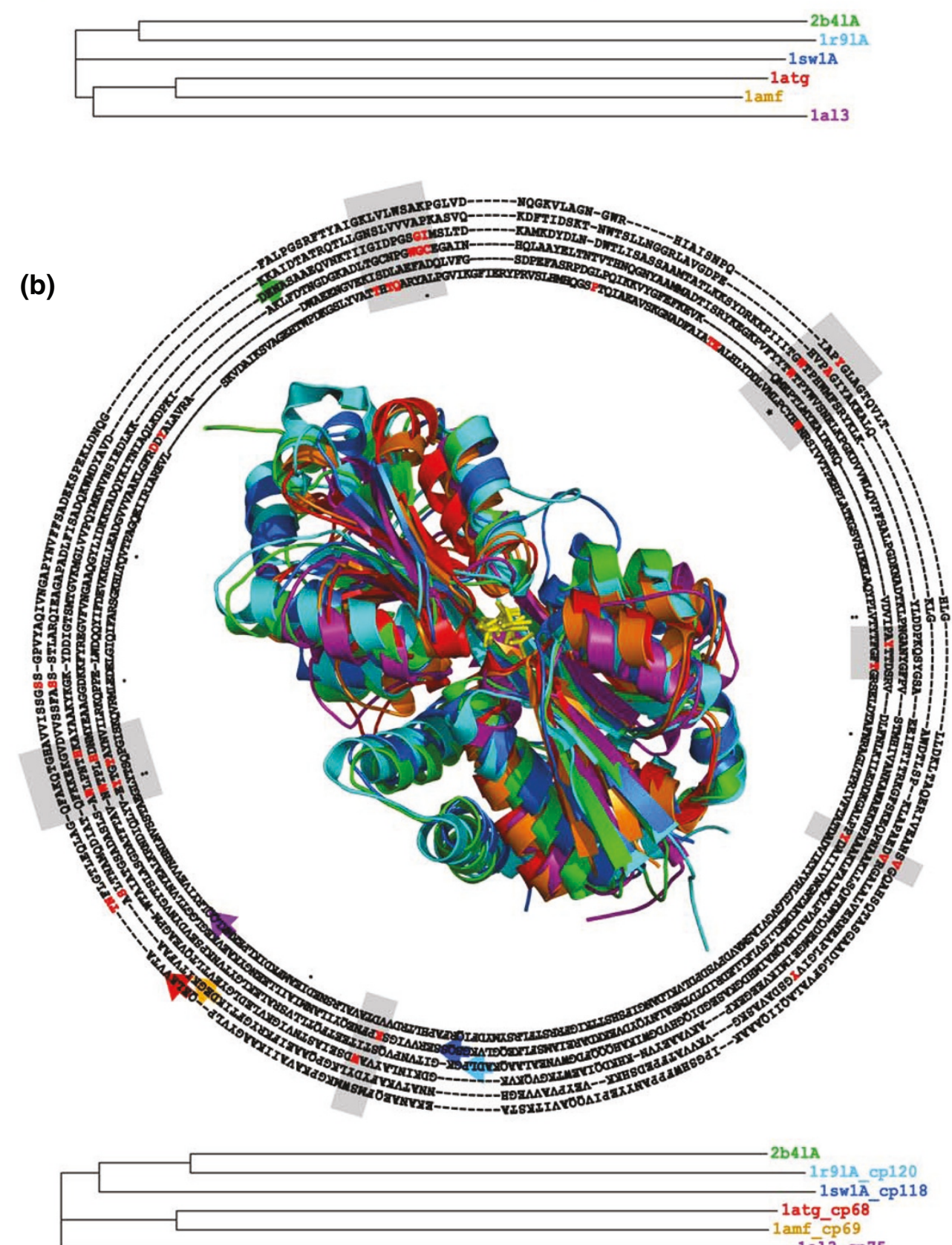

Figure 3 (see legend on following page) 
Figure 3 (see previous page)

A novel CP family detected by CPSARST. Entries 2b4IA ([PDB:2B4L], chain A), Ir9IA ([PDB:IR9L], chain A) and IswIA ([PDB: ISWI], chain A) are GBBPs. Entries Iatg ([PDB:IATG]) and Iamf ([PDB:| $\underline{\mid A M F]}$ ) are molybdate-binding proteins (MoBPs) and Ial3 ([PDB: $\mid$ AL3 $]$ ) is the cysteine regulon transcriptional activator CysB from Klebsiella aerogenes. Any pair of these proteins share $<24 \%$ sequence identity (calculated by FASTA [48]). (a) Multiple sequence alignment of these GBBPs, MoBPs and CysB does not well reveal their functional and evolutionary relationships. Residues interacting with the ligands [65-67] are colored red; they are rather scattered. GBBPs and MoBPs are basically ligand transporters while CysB is a transcriptional regulator; however, the phylogram tree built from this alignment correlates CysB and MoBPs into the same branch and the three GBBPs are separated into two branches; these evolutionary relationships do not agree with their functional relatedness. (b) Multiple circularly permuted sequence alignment and structural superimposition of these six proteins. The numbers after '_cp' following PDB entry IDs stand for the residue numbers of the new amino termini after circular permutations, which are indicated by colored arrows. The ligand-interacting residues are better clustered in this alignment (gray regions) and the phylogram tree agrees well with the functional relatedness. The image of the superimposed proteins shows that these proteins have similar overall structures and the positions of their ligand-binding pockets are conserved (ligands are shown as yellow stick models); the colors used in this image are the same as in the alignment text and phylogram tree. Structures shown in this report were all drawn by using PyMOL [68]. Multiple sequence alignments and the tree building were performed by Clustal W [69].

relationship between symmetric proteins is conditional upon the observation of a reasonable increase in sequence homology after the CP. For instance, B. subtilis thiaminase I [51] and Variovorax sp. Pal2 phosphonopyruvate hydrolase [52] are a pair of symmetric TIM-barrel proteins detected by CPSARST that superimpose well, with (alignment size, 151;

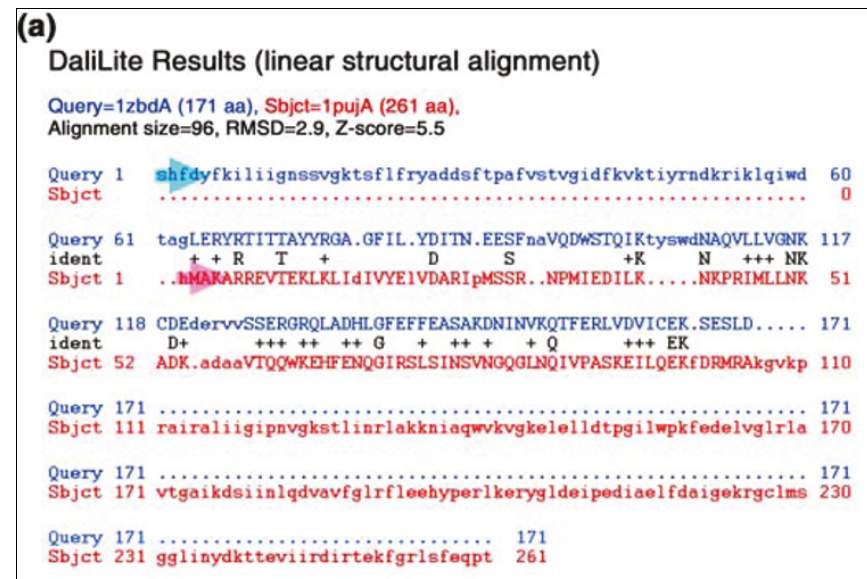

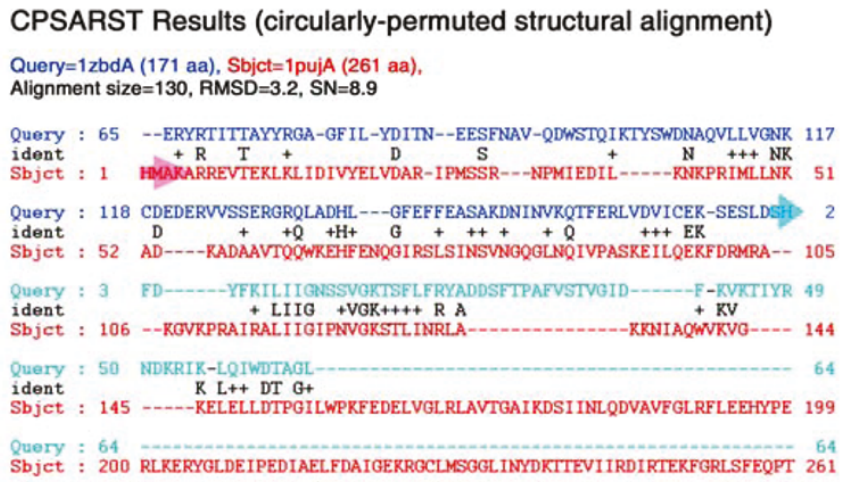
N-termini:

(b)
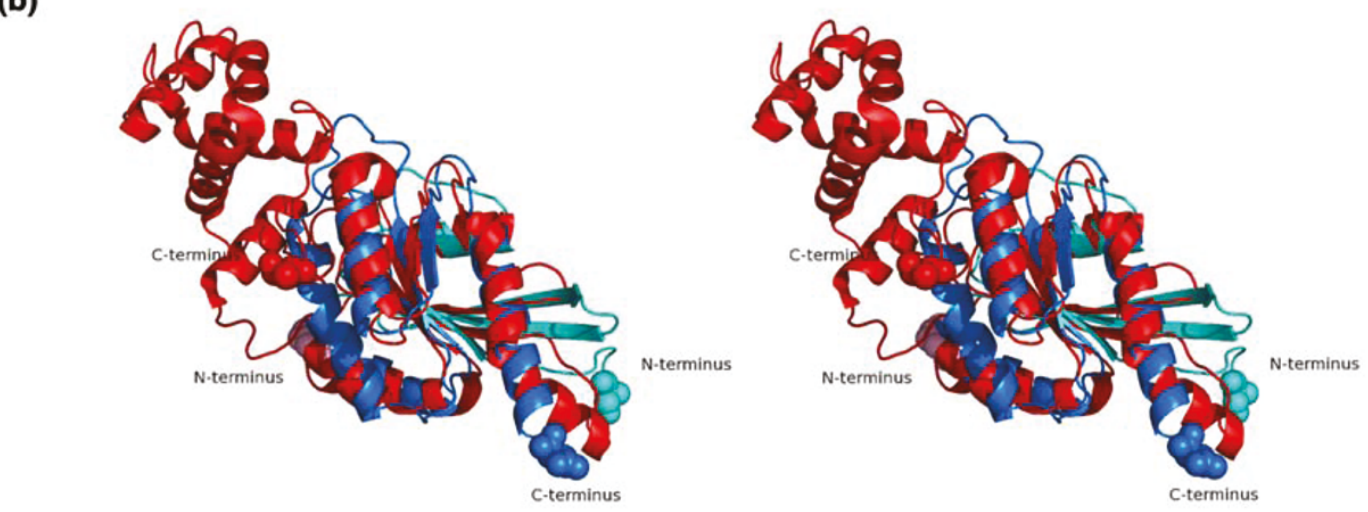

\section{Figure 4}

CP relationship between GTPase and hypothetical protein YlqF. Rab3A ([PDB:IZBD], chain A) is a small G protein with GTPase activity [49] while YlqF ([PDB:IPU]], chain A) is a conserved hypothetical protein from B. subtilis. (a) These two proteins can be structurally aligned by DALI [36] only partially (left); however, CPSARST detects their CP relationship (right). If the 64 residue amino-terminal region of Rab3A (in cyan text) is permuted to the carboxul terminus, it can be extensively aligned to YlqF with an RMSD of $3.2 \AA$ (right). The transparent cyan and pink arrows indicate the amino termini of Rab3A and YlqF, respectively. (b) The superimposition of Rab3A and YlqF made by CPSARST (cross-eye stereo view). Colors are the same as in (a). Residues shown as cyan/pink and blue/red spacefill models are the amino and carboxyl termini, respectively. 
Table 5

\begin{tabular}{|c|c|c|c|c|}
\hline No. & PDB entry/size & E-value & RMSD/Alignment size & Function \\
\hline I & $\underline{\mathrm{IZBD} / 203}$ & $4.00 \mathrm{E}-13$ & $3.17 / 130$ & Rabphilin-3A \\
\hline 2 & $\underline{\mathrm{IKY}} 2 / \mathrm{I} 82$ & $4.00 \mathrm{E}-13$ & $3.07 / 122$ & GTP-binding \\
\hline 3 & $\underline{2 \mathrm{~F} 7 \mathrm{~S}} / 217$ & $4.00 \mathrm{E}-13$ & $3.52 / 125$ & Ras-related protein Rab-27B protein YPT7P \\
\hline 4 & $\underline{2 N Z J / 175}$ & $8.00 \mathrm{E}-13$ & $2.94 / 123$ & GTP-binding protein REM I \\
\hline 5 & $\underline{\text { IT9|/207 }}$ & $9.00 \mathrm{E}-13$ & $3.06 / 123$ & Ras-related protein Rab-7 \\
\hline 6 & $\underline{\mathrm{IX} 3 \mathrm{~S} / 195}$ & $2.00 \mathrm{E}-12$ & $2.80 / 117$ & Ras-related protein Rab- 18 \\
\hline 7 & $\underline{\text { IYU9/I75 }}$ & $6.00 \mathrm{E}-12$ & $2.70 / 123$ & GTP-binding protein, GTPase domain \\
\hline 8 & $\underline{2 \mathrm{EWI}} / 20 \mathrm{I}$ & $6.00 \mathrm{E}-12$ & $2.74 / 128$ & Ras-related protein Rab-30 \\
\hline 9 & $\underline{2 G F 9 / 189}$ & $7.00 \mathrm{E}-12$ & $2.89 / 126$ & Ras-related protein Rab-3D \\
\hline 10 & IYVD/I69 & $8.00 \mathrm{E}-12$ & $2.12 / 123$ & Ras-related protein Rab-22A \\
\hline II & $\underline{|P U|} / 210$ & I.00E-II & $3.00 / 130$ & Probable GTP-binding protein engB \\
\hline 12 & $\underline{2052} / 200$ & I.00E-II & $2.92 / 127$ & Ras-related protein Rab-4B \\
\hline 13 & $\underline{\mathrm{IU} 8 Y} / 168$ & I.00E-II & $2.81 / 110$ & Ras-related protein Ral-A \\
\hline 14 & $\underline{\mathrm{H} U Q / / 64}$ & I.00E-II & $2.80 / 123$ & Rab5C, GTPase domain \\
\hline 15 & $\underline{2 H U P} / 201$ & $1.00 \mathrm{E}-1 \mathrm{I}$ & $3.11 / 129$ & Ras-related protein Rab-43 \\
\hline 16 & $\underline{\mid F Z Q} / 181$ & I.00E-II & $2.58 / 123$ & ADP-ribosylation factor-like protein 3 \\
\hline 17 & $\underline{20 C B} / / 80$ & $3.00 \mathrm{E}-\mathrm{II}$ & $2.78 / 121$ & Ras-related protein Rab-9B \\
\hline 18 & $101 \mathrm{~V} / 191$ & $4.00 \mathrm{E}-1 \mathrm{I}$ & $2.8 \mathrm{I} / \mathrm{I} 2 \mathrm{I}$ & Ras-related protein Rab-IIA \\
\hline 19 & $\underline{2 F N 4 / I 8 I}$ & $4.00 \mathrm{E}-1 \mathrm{I}$ & $3.11 / 129$ & Ras-related protein R-Ras \\
\hline 20 & $\underline{\mathrm{ZZOF} / \mathrm{I}} 79$ & $6.00 \mathrm{E}-11$ & $3.04 / 121$ & Rab|4, member Ras oncogene family \\
\hline
\end{tabular}

*YlqF ([PDB:IPU]], chain A) is a conserved hypothetical protein from B. subtilis. This structure was determined by the New York Structural Genomics Research Consortium (NYSGRC).

RMSD, $2.4 \AA$ ) or without (alignment size, 158; RMSD, $2.7 \AA$ ) CP. Their sequence identity rises from $10.1 \%$ to $24.3 \%$ upon $\mathrm{CP}$. As shown in Figure 5, their ligand-interacting residues are not well-aligned without CP while, for each protein, these functionally important residues can be aligned with physiochemically related amino acids on the other protein with $\mathrm{CP}$. Therefore, we suggest that this is a true CP case.

\section{Discussion \\ Detecting circular permutants with low sequence identities}

Generally speaking, although protein similarity search methods based on amino acid sequence alignments are much faster than those based on structural comparisons, they are less sensitive in detecting remote homology [53]. In the case of detecting $\mathrm{CP}$, sequence-based methods have met great challenges because of the evolutionary complexity and diversity of circular permutants. Except the post-translational modification model, all the other proposed mechanisms for CP involve at least two stages of genetic modifications in evolution (see Background), implying that the formation of $\mathrm{CP}$ may require a long period during which other common mutations (substitutions, insertions and deletions) can accumulate to such an extent that the circular permutants have much diverged from the parent protein in sequence. Therefore, sequence-based methods may be limited in identifying dis- tantly related CPs. For instance, Uliel et al. used an amino acid sequence-based heuristic algorithm to screen the entire Swiss-Prot database (version 34.0; approximately 80,000 proteins) and the Pfam database [54] for CP pairs, and identified only 32 cases [30]. However, in the same year, Jung and Lee [29] used a structure-based algorithm to survey a protein dataset (3,035 domains) collected from SCOP and reported that approximately $47 \%(1,433$ of 3,035$)$ of the domains each had at least one circular permutant. Furthermore, they discovered that less than $0.3 \%$ of the abundant symmetric CPs have $>30 \%$ sequence identities. Although this large difference is partially caused by the fact that Uliel et al. used more stringent criteria to identify $\mathrm{CP}$, it basically indicates that amino acid sequence-based methods can miss many distantly related CPs [34].

Among the CP candidate pairs detected by CPSARST in nrSCOP-90, 27.5\% can be considered as symmetric CPs (Table 4). Similar to the observation of Jung and Lee, few of these symmetric CPs (2.6\%) have sequence identities > 30\%. Furthermore, although $91 \%$ of the naturally occurring CP pairs listed in Table 2 have sequence identities $\leq 20 \%$, CPSARST shows good performance when compared with other structure-based methods. These data demonstrate that CPSARST is able to detect CPs with low sequence identities. 
(a)

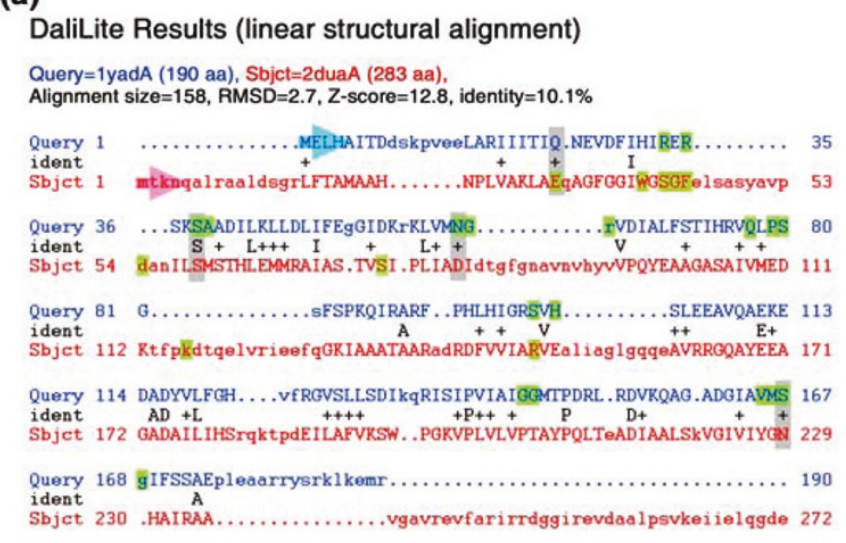

$\begin{array}{llll}\text { Query } & 190 & \ldots \ldots \ldots \ldots & 190 \\ \text { Sbjct } & 273 & \text { rmavearyik } 283\end{array}$

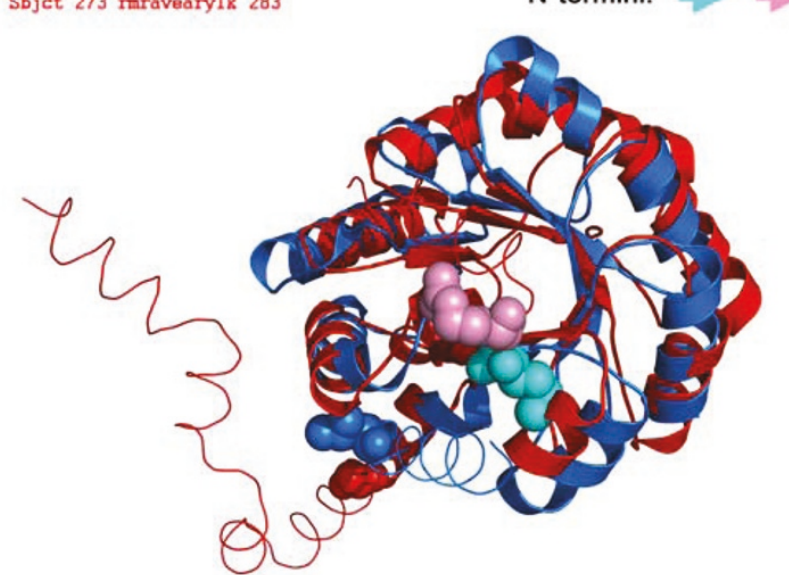

(b)
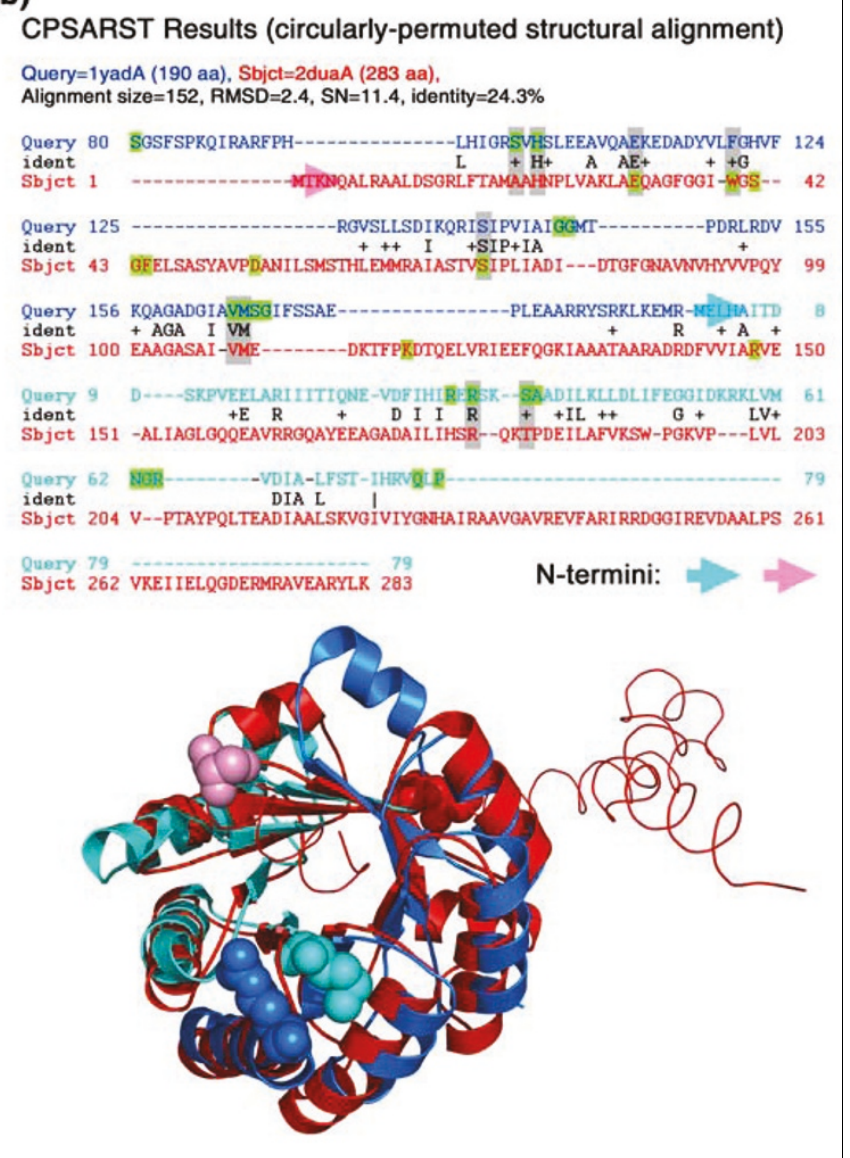

Figure 5

Symmetric CP with significant sequence clues. Proteins with symmetric structure may have symmetric CPs [29]. B. subtilis thiaminase I ([PDB:IYAD]) [5I] and Variovorax sp. Pal2 phosphonopyruvate hydrolase ([PDB:2DUA]) [52] shown here are symmetric TIM-barrel proteins. Although their structures can be well-aligned both by linear and CP alignments, significant sequence conservation is observed only in the latter. (a) Linear alignment performed by DALI [36]. The upper text demonstrates that the sequence identity calculated from these structurally aligned residues is $10.1 \%$. Ligand-interacting residues in both proteins are highlighted green; four of them are aligned with identical or physiochemically similar amino acids (gray highlighted strips). The lower image is the superimposition of these two structures. Terminal unaligned regions are shown as ribbons to make the spatial closeness of the termini more easily observable. In this linear alignment, the amino termini of the two proteins are close to each other, as are the carboxyl termini. (b) CP relationship detected by CPSARST. After CP, the sequence identity significantly rises to $24.3 \%$ and there are nine ligand-interacting residues aligned with identical or similar amino acids. The amino- and carboxy-terminal halves of lyadA bounded by the putative CP site are colored cyan and blue, respectively. The orientation of lyadA in the superimposed image is the same as that in (a). In this CP alignment, the amino and carboxyl termini of the two proteins are separated, a feature of symmetric CP.

\section{Speed improvements}

In most cases, it is not easy to achieve high accuracy and speed simultaneously for a database search method; instead, some compromising balance is usually reached. Judging from the fact that using previous structure-based CP-detecting methods such as SAMO to search the current PDB requires more than 15,000 minutes [34] per query, it is reasonable that speed should be weighted more than accuracy in the field of CP searching, especially in this post-genomic era when the amount of protein structural data is increasing rapidly. CPSARST has been shown to achieve accuracy substantially higher than sequence-based UFAU (Figure 2) and comparable to structure-based SAMO (Tables 2 and 3); as to the speed, it can scan 52,800 database proteins per minute
(Table 4), approximately 4 and 8,824 times faster than UFAU and SAMO, respectively. This improvement in speed is achieved by two features: it transforms three-dimensional information of protein structures into one-dimensional text strings and, thus, converts structural comparison problems into text sequence alignment problems, which can be solved much more rapidly; and, in both the screening and refinement stages, CPSARST does not stick to the absolute qualities of the alignments. By focusing on the relative qualities between two rounds of alignments, it can rapidly sieve out useful information. We call this strategy 'double filter-andrefine'. Here we propose that it is efficient, flexible and applicable to other biological research fields, especially where the data analyses require large-scale computational power. 
The prevalence and definition of circular permutation Previous studies have made conflicting conclusions; some presumed that $\mathrm{CP}$ is rare in nature $[6,14,30]$ - approximately $5 \%$ as indicated by Vogel and Morea [14] - while others supposed that CP is frequent $[1,29,34]$ - approximately $47 \%$ as estimated by Jung and Lee [29]. In our observation, studies based on structural analyses usually discovered more CPs than sequence-based ones; besides, studies that consider the whole protein as the unit that undergoes $\mathrm{CP}$ would conclude that $\mathrm{CP}$ is rare whereas those viewing the domain as the unit that undergoes $\mathrm{CP}$ would suggest $\mathrm{CP}$ to be frequent.

As we have discussed, it is reasonable that more cases of CP are detected by structural comparison than by amino acid sequence alignment. However, although proteins with similar structures are usually functionally related [55], when a pair of structurally and functionally similar proteins share extremely low sequence identity, we still cannot exclude the possibility that they are just the products of convergent evolution [5658 ] and do not share the same origin. In the case of identifying $\mathrm{CP}$, it is noteworthy that even if a pair of proteins shows a high extent of CP topologically, it does not directly mean that an evolutionary $\mathrm{CP}$ event has indeed taken place. Therefore, we argue that detecting $\mathrm{CP}$ only by structure would result in too many false positives when judged from the point of view of molecular evolution. This is why we have set up a useradjustable sequence identity filter in the web service of CPSARST [41] (see Materials and methods). When this filter was not enabled, the prevalence of CP estimated by CPSARST was $12.6-17.6 \%$ (see Results). When we considered that a real $\mathrm{CP}$ should have a higher sequence identity in the CP alignment than in the linear alignment, around one-fourth of the candidate pairs counted in Table 4 was filtered out, lowering the estimated prevalence of $\mathrm{CP}$ to $9.0-13.0 \%$.

The fact that the frequency of CP estimated by CPSARST is only one-third of that estimated by Jung and Lee [29] is probably because of the more stringent criteria used by CPSARST. We set the RMSD cutoff as $5 \AA$, the CP score threshold as 0.2 and the least permutation size as $20 \%$ for a pair of proteins to be considered as CP candidates; similar criteria were not seen in the report of Jung and Lee. Also, considering their methodology, there is a large likelihood that proteins containing repeats and duplications are regarded as CPs, many of which have been treated as false cases by Uliel et al. [30] and us (see Materials and methods). When we loosened the criteria to $6 \AA$ (RMSD cutoff), 0.1 (CP score threshold) and 10\% (least permutation size), and did not filter out proteins containing repeats, the CP prevalence estimated by CPSARST was 34.7$36.7 \%$ (see Additional data file 5 for statistics), similar to Jung and Lee's estimation. However, since they did not provide any supplementary list of their $\mathrm{CP}$ candidates, we are unable to check our speculation.

To our knowledge, all the currently available CP-detecting methods are more sensitive to global CP (the unit undergoing
$\mathrm{CP}$ is the whole protein) than partial $\mathrm{CP}$ (the $\mathrm{CP}$ is within a region of the protein), as is CPSARST. To detect partial CP, domain databases such as SCOP and Pfam are usually used as the target databases instead of the PDB and Swiss-Prot. Although considering the domain as the unit undergoing $\mathrm{CP}$, that is, partial CP, can identify more candidates (as shown in Table 4), some scientists have argued that these cases should be considered as 'swaps' rather than CPs [30]. This controversy is another cause of the conflicting conclusions about the prevalence of $\mathrm{CP}$.

To sum up, despite the conflicting conclusions made by previous studies, there seem to be rational explanations for this situation. We suppose that the identification of $\mathrm{CPs}$ requires a precise definition of $\mathrm{CP}$ depending on the purpose of the study. In our opinion, if evolutionary importance and mechanisms are concerned, global $\mathrm{CP}$ with reasonable sequence identity limitation will be suitable, while partial $\mathrm{CP}$ without limitation of sequence identity in the definition may help scientists to discover novel functional relationships among proteins and to reveal the principles of protein folding.

\section{Possible applications of CPSARST}

The performance of CPSARST suggests that it is an efficient approach to the detection of CPs in large protein structural datasets; routine bank-against-bank searches are thus achievable. The multiple indexes produced by CPSARST, for example, the structural similarity score, statistically meaningful E-value, sequence identity, alignment size, RMSD and CP score, are beneficial to develop automated procedures such as a functional assignment system for novel hypothetical proteins. Also, information retrieved by bank-against-bank searches can be organized into a CP database.

Since the first observation of CP in plant lectins [5], many natural and artificial cases have been studied and several CP detecting methods have been developed; however, there is still no CP database and no standard procedure for evaluating $\mathrm{CP}$ detection methods. We suppose that a well-organized CP database will help move this field forward. It could provide a standard for the evaluations of CP-related programs, such as CP search tools and predictors of viable CP sites [59], and provide information to reveal the evolutionary mechanisms of CP.

CP has been applied to X-ray crystallography [22], modification of enzymes [15], creation of novel fusion proteins $[25,28]$, and construction of protein switches and sensors $[26,27]$. All these applications depend on a proper choice of position to create CP. A CP database offering plenty of materials for the discovery of the rules by which Nature selects CP sites should be advantageous to the technical applications of CP.

Although interesting, there is still much uncertainty about the evolutionary mechanisms and importance of CP $[6,18,29,30]$. 
Weiner et al. [6o] have proposed that the frequency of incomplete or intermediate CP may help determine the major mechanism of CP. The 'double filter-and-refine' strategy of CPSARST is very flexible. With extended boundary criteria, CPSARST can specifically detect incomplete or intermediate CP. The ability of CPSARST to perform rapid bank-againstbank searches by structural comparisons gives it the potential to reveal how, why and to what extent Nature achieves protein evolutionary and functional diversity by using CP.

\section{Conclusion}

We have developed an efficient circular permutation search method, CPSARST, which linearly encodes protein structures as text strings and achieves a structural similarity searching speed thousands of times as high as related algorithms. When tested with engineered CPs, CPSARST successfully retrieved all the natural proteins with accurate permutation site predictions. Its ability to identify natural CPs is also comparable to other structure-based CP-detecting methods. Its high efficiency makes routine database surveys and bank-againstbank searches achievable. After all-against-all searches of non-redundant PDB and SCOP, we have found that most candidate CP pairs share sequence identity $<20 \%$, explaining why previous sequence-based $\mathrm{CP}$-detecting methods have identified much less CP cases than structure-based algorithms. Based on these search results, we have suggested that the identification of CPs requires a suitable definition of CP depending on the purpose of the study. If global CP with reasonable sequence identity limitation is considered as true $\mathrm{CP}$, the prevalence of $\mathrm{CP}$ in protein structural databases is estimated to be $16 \%$ by CPSARST, whereas the prevalence of partial CP without limitation of sequence identity in the definition is estimated to be $36 \%$. Several new CP cases have been detected and reported here, inclusive of a novel CP family consisting of microbial GBBPs, molybdate-binding proteins and a cysteine regulon transcriptional activator. In this post-genomics era, when the amount of protein structural data is increasing exponentially, CPASRST can provide a new way to rapidly detect novel relationships among proteins and help to reveal how Nature achieves protein evolutionary and functional diversity by using CP. Its web service and standalone Java program are available at [41].

\section{Materials and methods}

All the developments and experiments were performed on an IBM e-server 336 machine with dual 3.2 GHz Intel processors, 1 GB RAM and linux operating system.

\section{Linear encoding of protein structures}

CPSARST describes three-dimensional protein structures as one-dimensional strings by using a RST algorithm [40]. The torsion angles $(\phi, \delta)$ of a number of proteins were plotted onto a $10^{\circ} \times 10^{\circ}$ dissected RM map. The 1,296 cells on this map were then clustered into 22 groups by nearest-neighbor clus- tering [61] based on their spot numbers and angular distances. These groups were assigned a set of English letters called 'Ramachandran codes'. Coordinates of a protein structure could be accordingly transformed into a text string. The scoring matrix for these codes was produced by using a 'regenerative approach' [40]. This linear encoding system converts complicated and time-consuming structural comparison problems into sequence comparisons, which can be done very rapidly. It has been applied to protein structural similarity searching and achieved speeds hundreds of thousands of times higher than CE with an acceptable compromise of accuracy [40]. The structural string generated by RST is different from the amino acid sequence in nature; therefore, we termed it 'Ramachandran sequence' or 'Ramachandran string'.

\section{Generation and analyses of random circular permutants}

A hundred polypeptide sequences each longer than 100 residues and sharing < 40\% sequence identities were randomly selected from the PDB to perform in silico circular permutations. Regular mutations, i.e. substitutions, insertions and deletions, were introduced in the ratio 150:1:1 to generate random CPs, resulting in 100 levels of decreasing sequence identities/similarities for every polypeptide sequence. The collection of these computer-generated random CPs is called the RCP dataset.

The substitution rates of various amino acids used to generate the RCP dataset were calculated by analyzing a large number of multiple alignment blocks, the sequences of which shared $<45 \%$ identity, as described previously [62]. Since every sequence in the RCP dataset was evolved independently to avoid any possible bias, we supposed that it is suitable for the evaluation of CP detection methods. RCP has two subsets, the identity subset and similarity subset, each containing 10,000 CP pairs (100 parent sequences $\times 100$ circular permutants). They are listed in Additional data file 6.

Comparisons between each parent sequence and its CPs in the identity subset of the RCP dataset were performed by the traditional heuristic method blast [45]. Two parameters were monitored to assess the performance: the percentage of cases in which the exact permutation site was retrieved; and the average percentage distance of the found permutation site to the exact one (see Results). Another two parameters were monitored to optimize the filter for RM sequence searches: the ratio of similarity scores and the negative logarithm in base $10\left(-\log _{10}\right)$ of the E-value ratios, before and after the duplication of query sequences (see Additional data file 7 for the results). We found that all the score ratios are equal to or higher than 1, indicating that when the sequence of a CP is duplicated (DL), it always aligns to its parent sequence better than the normal length (NL). As to the E-value ratios, that is, $-\log _{10}\left(E-\right.$ value $_{D L} / E$-value $\left.{ }_{N L}\right)$, approximately $80 \%$ of them are larger than 2, which stands for a $10^{2}$-fold improvement of the 
significance of the similarity score after duplicating the query sequence (see Results for detailed information about E-values).

\section{Screening of circular permutant candidates}

It has been supposed that using heuristic methods like blast to search for CPs is difficult because an unambiguous reconstruction of the alignment results is problematic [38]. CPSARST, however, overcomes this problem by duplicating the query structure, doing two rounds (with and without the duplication) of database searches, and analyzing the results mutually. The hits with improved alignment qualities are picked as CP candidates, the permutation sites of which can be easily determined from the alignment results of duplicated sequences. In the screening stage, the search results of RM strings were filtered with simple criteria referring to previous studies and our experimental results on RCP amino acid sequences mentioned above. The permutation site should be at between $20 \%$ and $80 \%$ along the length of the query protein, ensuring a significant permutation size (20\%). It has been supposed that a tiny permutation size is unlikely a real $\mathrm{CP}$ [39]. The size of the candidate could be different from that of the query protein by at most $50 \%$ because proteins of very different sizes are improbable candidates for CPs [38]. The similarity score of the duplicated query string $\left(\right.$ Score $_{D L}$ ) should be higher than that of the normal query string $\left(\right.$ Score $\left._{N L}\right)$, and the $-\log _{10}$ value of the E-value ratio should be larger than -0.5 (see Additional data file 7 for detailed information about these settings):

$$
\begin{gathered}
\frac{\text { Score }_{D L}}{\text { Score }_{N L}}>1 \\
-\log _{10}\left(\frac{\text { Evalue }_{D L}}{\text { Evalue }_{N L}}\right)>-0.5
\end{gathered}
$$

\section{Refinement of the search results}

The refinement of search results of RM sequences were performed by FAST, an accurate structural alignment algorithm [40,63] and a CP scoring scheme developed by Vesterstrom and Taylor [39], following these steps. Step 1: for each candidate, the putative permutation site is parsed from the alignment result of the duplicated query string. Step 2: performing two rounds of FAST structural alignments. The first round is a normal linear alignment. In the second round, the circularly permuted alignment, the PDB file of the query structure was manipulated by exchanging the amino- and carboxy-terminal halves according to the putative permutation site so that FAST will do the structural alignment 'backside first'. Step 3: if the FAST alignment size after CP is no larger than $50 \%$ of the smaller size of the query and subject proteins, it is screened out. Step 4: the RMSD cutoff of the CP alignment is set as $5 \AA$. Step 5: in order to differentiate true CP from protein with internal repeats or duplications, two criteria have been set: the alignment size of the CP alignment should be larger than that of the linear alignment; and the FAST simi- larity score [63] or TOP score [43] (see formula (2)) calculated from the CP alignment should gain at least $25 \%$ improvement over the linear alignment. Step 6: the CP score [39] was calculated from the aligned positions by FAST. It has a theoretical minimum value of -1 (a completely linear alignment) and a maximum value of 1 (a perfect $\mathrm{CP}$ ). Although Vesterstrom and Taylor suggested that an alignment with this $\mathrm{CP}$ score higher than 0.25 can be considered as a significant $\mathrm{CP}$, we find that 0.2 is still suitable in our multi-filter system. Step 7: the putative CP site is refined by parsing the output of FAST structural alignment.

\section{Pair-wise circularly-permuted structural alignments}

The procedure of the database search tool CPSARST can be simplified to perform pair-wise structure alignments as follows. First, transform the query and subject protein structures into RM sequences Q and S, respectively. Second, duplicate Q string to QQ, and align it to S. Third, find the best local alignment and trace it back to the 'start point', which is the putative permutation site. For example, if in the best local alignment, the fragment between residues $q_{1}$ and $q_{2}$ of $\mathrm{Q}$ is aligned to the fragment between $s_{1}$ and $s_{2}$ of $S$, then the permutation site of $\mathrm{Q}$ will be traced back to $q_{1}-s_{1}+1$. Fourth, introduce a $\mathrm{CP}$ into the query structure according to the putative CP site. Compare this new structure with the subject protein by using an accurate structural alignment algorithm mentioned above.

\section{Implementation}

CPSARST basically works on the structurally meaningful RM strings transformed by RST; however, since there have been many errors and inconsistencies reported in PDB entries [64], a few polypeptides (approximately 2\%) cannot be successfully transformed into RM strings. Therefore, in the implementation of CPSARST, we have added two extra rounds of amino acid sequence alignment searches, one by the normal length and the other by the duplicated sequence, prior to the RM string searches. Besides, the sequence homology filter can be enabled to guarantee a higher evolutionary significance of the search results (see Discussion), and several parameters are adjustable by the users according their needs or the property of materials.

\section{Word size and gap penalties}

These are traditional parameters used by sequence alignment search tools such as BLAST [45] and FASTA [48]. For CPSARST, a smaller word size can provide a more accurate determination of the CP site while taking more running time. In our experience, lower gap penalties can give CPSARST higher sensitivity, although there is a trade-off for running time, too. Generally speaking, these parameters have only minor effects on the performance.

\section{Permutation size limit and circular permutation score threshold} It has been supposed that a tiny permutation size is unlikely a real CP [39], but there is yet no common conclusion made for 
the generally suitable permutation size limit. Setting a large limit ensures that CPSARST identifies unambiguous CP relationships; however, novel cases can thus be missed. If the query protein is large enough, for example, $>150$ residues, a small size limit such as 10\% may still work well, but we would like to suggest a $15 \%$ limit for general situations. The CP score threshold has similar effects on the search quality of CPSARST to the permutation size limit (see Results and Materials and methods for further information).

\section{RMSD cutoff and structural similarity improvement filter}

Closer-related protein structures will have a lower RMSD when superimposed. This is also true for CPs. This cutoff can be used as a basic quality control in the same way as other conventional structural comparison tools. The normalized structural similarity score of FAST [63] is another basic quality control. Candidate pairs without enough improvement in structural similarity after CP can be screened out.

Examples of practical settings for these parameters can be found in Additional data file 8. CPSARST is available at [41].

\section{Abbreviations}

CP, circular permutation; CPs, circular permutants; CPSARST, Circular Permutation Search Aided by Ramachandran Sequential Transformation; DL, duplicated; GBBP, glycine betaine-binding protein; NL, normal length; PDB, Protein Data Bank; RM, Ramachandran; RCP, random circular permutation; RMSD, root mean square distance; RST, Ramachandran sequential transformation.

\section{Authors' contributions}

WCL designed and carried out this study and drafted the manuscript. PCL conceived the study, participated in its design and helped to draft the manuscript.

\section{Additional data files}

The following additional data are available with the online version of this paper. Additional data file 1 lists the nrPDB-90 dataset, the 90\% sequence identity subset of the PDB (January 2007). Additional data file 2 lists the nrSCOP-90 dataset, the $90 \%$ sequence identity subset of SCOP (v.1.71). Additional data file 3 is a table listing candidate CP pairs in the nrPDB90 dataset detected by CPSARST with RMSD $\leq 3.5 \AA$ A. Additional data file 4 is a list of the structural neighbors of the hypothetical protein YlqF in PDB retrieved by DALI [50]. Additional data file 5 is a table showing statistical results of protein structural database searches with broad criteria by CPSARST. Additional data file 6 lists the RCP dataset, a collection of 20,00o in silico random CPs. Additional data file 7 is a plot summarizing the score and E-value ratios calculated from the RCP dataset. Additional data file 8 is a list of the parameter settings used throughout this article.

\section{Acknowledgements}

This work was supported by the National Science Council, Taiwan, ROC (NSC grant numbers: 95-3।I2-B-007-006 and 96-3।I2-B-007-006). We thank the authors of the BLAST and FAST algorithms, which were extensively used in this study.

\section{References}

I. Jeltsch A: Circular permutations in the molecular evolution of DNA methyltransferases. J Mol Evol 1999, 49:16I-164.

2. Weiner J 3rd, Thomas G, Bornberg-Bauer E: Rapid motif-based prediction of circular permutations in multi-domain proteins. Bioinformatics 2005, 21 :932-937.

3. Tsai LC, Shyur LF, Lee SH, Lin SS, Yuan HS: Crystal structure of a natural circularly permuted jellyroll protein: I,3-I,4-beta-Dglucanase from Fibrobacter succinogenes. J Mol Biol 2003, 330:607-620.

4. Ribeiro EA Jr, Ramos $\mathrm{CH}$ : Circular permutation and deletion studies of myoglobin indicate that the correct position of its $\mathrm{N}$-terminus is required for native stability and solubility but not for native-like heme binding and folding. Biochemistry 2005, 44:4699-4709.

5. Cunningham BA, Hemperly JJ, Hopp TP, Edelman GM: Favin versus concanavalin A: circularly permuted amino acid sequences. Proc Natl Acad Sci USA 1979, 76:3218-3222.

6. Lindqvist $Y$, Schneider $G$ : Circular permutations of natural protein sequences: structural evidence. Curr Opin Struct Biol 1997, 7:422-427.

7. Murzin AG: Probable circular permutation in the flavin-binding domain. Nat Struct Biol 1998, 5:101.

8. Castillo RM, Mizuguchi K, Dhanaraj V, Albert A, Blundell TL, Murzin AG: A six-stranded double-psi beta barrel is shared by several protein superfamilies. Structure 1999, 7:227-236.

9. Polekhina G, Board PG, Gali RR, Rossjohn J, Parker MW: Molecular basis of glutathione synthetase deficiency and a rare gene permutation event. EMBO J 1999, 18:3204-3213.

10. Bujnicki JM: Sequence permutations in the molecular evolution of DNA methyltransferases. BMC Evol Biol 2002, 2:3.

II. Jung J, Lee $B$ : Protein structure alignment using environmental profiles. Protein Eng 2000, 13:535-543.

12. Antcheva N, Pintar A, Patthy A, Simoncsits A, Barta E, Tchorbanov B, Pongor S: Proteins of circularly permuted sequence present within the same organism: the major serine proteinase inhibitor from Capsicum annuum seeds. Protein Sci 200I, 10:2280-2290.

13. Goldenberg DP, Creighton TE: Circular and circularly permuted forms of bovine pancreatic trypsin inhibitor. J Mol Biol 1983, 165:407-413.

14. Vogel C, Morea V: Duplication, divergence and formation of novel protein topologies. Bioessays 2006, 28:973-978.

15. Qian Z, Lutz S: Improving the catalytic activity of Candida antarctica lipase B by circular permutation. J Am Chem Soc 2005, I 27:13466-I3467.

16. Anantharaman V, Koonin EV, Aravind L: Regulatory potential, phyletic distribution and evolution of ancient, intracellular small-molecule-binding domains. J Mol Biol 200I, 307:127I-1292.

17. Todd $A E$, Orengo CA, Thornton JM: Plasticity of enzyme active sites. Trends Biochem Sci 2002, 27:419-426.

18. Bulaj G, Koehn RE, Goldenberg DP: Alteration of the disulfidecoupled folding pathway of BPTI by circular permutation. Protein Sci 2004, 13:1 I82-1196.

19. Heinemann U, Hahn M: Circular permutations of protein sequence: not so rare? Trends Biochem Sci 1995, 20:349-350.

20. Li L, Shakhnovich El: Different circular permutations produced different folding nuclei in proteins: a computational study. J Mol Biol 200I, 306:121-132.

21. Chen J, Wang J, Wang W: Transition states for folding of circular-permuted proteins. Proteins 2004, 57:153-I7I.

22. Schwartz TU, Walczak R, Blobel G: Circular permutation as a tool to reduce surface entropy triggers crystallization of the signal recognition particle receptor beta subunit. Protein Sci 2004, 13:2814-2818. 
23. Anand B, Verma SK, Prakash B: Structural stabilization of GTP. binding domains in circularly permuted GTPases: implications for RNA binding. Nucleic Acids Res 2006, 34:2 I 96-2205.

24. Gebhard LG, Risso VA, Santos J, Ferreyra RG, Noguera ME, Ermacora MR: Mapping the distribution of conformational information throughout a protein sequence. J Mol Biol 2006, 358:280-288.

25. Kojima M, Ayabe K, Ueda $\mathrm{H}$ : Importance of terminal residues on circularly permutated Escherichia coli alkaline phosphatase with high specific activity. J Biosci Bioeng 2005, I 00:197-202.

26. Ostermeier $M$ : Engineering allosteric protein switches by domain insertion. Protein Eng Des Sel 2005, I 8:359-364.

27. Galarneau A, Primeau M, Trudeau LE, Michnick SW: Beta-lactamase protein fragment complementation assays as in vivo and in vitro sensors of protein protein interactions. Nat Biotechnol 2002, 20:619-622.

28. Baird GS, Zacharias DA, Tsien RY: Circular permutation and receptor insertion within green fluorescent proteins. Proc Natl Acad Sci USA 1999, 96: I I 24 I-I I 246.

29. Jung J, Lee B: Circularly permuted proteins in the protein structure database. Protein Sci 2001, 10:188|-1886.

30. Uliel S, Fliess A, Unger R: Naturally occurring circular permutations in proteins. Protein Eng 2001, I 4:533-542.

31. Carrington DM, Auffret A, Hanke DE: Polypeptide ligation occurs during post-translational modification of concanavalin $A$. Nature 1985, 3 I 3:64-67.

32. Ponting CP, Russell RB: Swaposins: circular permutations within genes encoding saposin homologues. Trends Biochem Sci 1995, 20:179-180

33. Peisajovich SG, Rockah L, Tawfik DS: Evolution of new protein topologies through multistep gene rearrangements. Nat Genet 2006, 38: 168-174.

34. Chen L, Wu LY, Wang Y, Zhang S, Zhang XS: Revealing divergent evolution, identifying circular permutations and detecting active-sites by protein structure comparison. BMC Struct Biol 2006, 6: 18.

35. Russell RB, Ponting CP: Protein fold irregularities that hinder sequence analysis. Curr Opin Struct Biol 1998, 8:364-37I.

36. Holm L, Sander C: Protein structure comparison by alignment of distance matrices. J Mol Biol 1993, 233: 123-138.

37. Shindyalov IN, Bourne PE: Protein structure alignment by incremental combinatorial extension (CE) of the optimal path. Protein Eng 1998, I I:739-747.

38. Uliel S, Fliess A, Amir A, Unger R: A simple algorithm for detecting circular permutations in proteins. Bioinformatics 1999 I5:930-936.

39. Vesterstrom J, Taylor WR: Flexible secondary structure based protein structure comparison applied to the detection of circular permutation. I Comput Biol 2006, 13:43-63.

40. Lo WC, Huang PJ, Chang CH, Lyu PC: Protein structural similarity search by Ramachandran codes. BMC Bioinformatics 2007, 8:307.

41. CPSARST - Circular Permutation Search Aided by Ramachandran Sequential Transformation [http:// sarst.life.nthu.edu.tw/cpsarst]

42. Berman HM, Westbrook J, Feng Z, Gilliland G, Bhat TN, Weissig H, Shindyalov IN, Bourne PE: The Protein Data Bank. Nucleic Acids Res 2000, 28:235-242

43. Lu G: Top: A new method for protein structure comparisons and similarity searches. J Appl Crystallogr 2000, 33:|76-|83.

44. Chandonia JM, Hon G, Walker NS, Lo Conte L, Koehl P, Levitt M, Brenner SE: The ASTRAL Compendium in 2004. Nucleic Acids Res 2004, 32:D189-192.

45. Altschul SF, Madden TL, Schaffer AA, Zhang J, Zhang Z, Miller W, Lipman DJ: Gapped BLAST and PSI-BLAST: a new generation of protein database search programs. Nucleic Acids Res 1997, 25:3389-3402

46. Altschul SF, Gish W: Local alignment statistics. Methods Enzymol 1996, 266:460-480.

47. Altschul SF, Gish W, Miller W, Myers EW, Lipman DJ: Basic local alignment search tool. J Mol Biol 1990, 21 5:403-4I0.

48. Pearson WR: Flexible sequence similarity searching with the FASTA3 program package. Methods Mol Biol 2000, I32: I 85-2 I9.

49. Ostermeier C, Brunger AT: Structural basis of Rab effector specificity: crystal structure of the small $G$ protein Rab3A complexed with the effector domain of rabphilin-3A. Cell 1999 , 96:363-374

50. The Dali Server [http://www.ebi.ac.uk/dali/]

5I. Toms AV, Haas AL, Park JH, Begley TP, Ealick SE: Structural char- acterization of the regulatory proteins TenA and Tenl from Bacillus subtilis and identification of TenA as a thiaminase II. Biochemistry 2005, 44:2319-2329.

52. Chen CC, Han Y, Niu W, Kulakova AN, Howard A, Quinn JP, Dunaway-Mariano D, Herzberg $O$ : Structure and kinetics of phosphonopyruvate hydrolase from Variovorax sp. Pal2: new insight into the divergence of catalysis within the PEP mutase/isocitrate lyase superfamily. Biochemistry 2006, 45: I | 49 |- I I 504.

53. Sauder JM, Arthur JW, Dunbrack RL Jr: Large-scale comparison of protein sequence alignment algorithms with structure alignments. Proteins 2000, 40:6-22.

54. Bateman A, Birney E, Durbin R, Eddy SR, Howe KL, Sonnhammer EL: The Pfam protein families database. Nucleic Acids Res 2000 , 28:263-266

55. Zuckerkandl E, Pauling L: Evolutionary divergence and convergence in proteins. In Evolving Genes and Proteins New York: Academic Press; 1965:97-166.

56. Krishna SS, Grishin NV: Structurally analogous proteins do exist! Structure 2004, I 2: I I 25- I I 27

57. Theobald DL, Wuttke DS: Divergent evolution within protein superfolds inferred from profile-based phylogenetics. J Mol Biol 2005, 354:722-737

58. Ausiello G, Peluso D, Via A, Helmer-Citterich M: Local comparison of protein structures highlights cases of convergent evolution in analogous functional sites. BMC Bioinformatics 2007 , 8(Suppl I):S24.

59. Paszkiewicz KH, Sternberg MJ, Lappe M: Prediction of viable circular permutants using a graph theoretic approach. Bioinformatics 2006, 22: 1353-1358.

60. Weiner J 3rd, Bornberg-Bauer E: Evolution of circular permutations in multidomain proteins. Mol Biol Evol 2006, 23:734-743.

6I. Jain AK, Dubes RC: Algorithms for Clustering Data New Jersey: Prentice Hall; 1988.

62. Henikoff S, Henikoff JG: Amino acid substitution matrices from protein blocks. Proc Natl Acad Sci USA 1992, 89:10915-10919.

63. Zhu J, Weng Z: FAST: a novel protein structure alignment algorithm. Proteins 2005, 58:618-627.

64. Westbrook J, Feng Z, Jain S, Bhat TN, Thanki N, Ravichandran V, Gilliland GL, Bluhm WF, Weissig H, Greer DS, Bourne PE, Berman HM: The Protein Data Bank: unifying the archive. Nucleic Acids Res 2002, 30:245-248.

65. Horn C, Sohn-Bosser L, Breed J, Welte W, Schmitt L, Bremer E: Molecular determinants for substrate specificity of the ligand-binding protein OpuAC from Bacillus subtilis for the compatible solutes glycine betaine and proline betaine. J Mol Biol 2006, 357:592-606.

66. Schiefner A, Breed J, Bosser L, Kneip S, Gade J, Holtmann G, Diederichs K, Welte W, Bremer E: Cation-pi interactions as determinants for binding of the compatible solutes glycine betaine and proline betaine by the periplasmic ligand-binding protein ProX from Escherichia coli . I Biol Chem 2004, 279:5588-5596.

67. Schiefner A, Holtmann G, Diederichs K, Welte W, Bremer E: Structural basis for the binding of compatible solutes by ProX from the hyperthermophilic archaeon Archaeoglobus fulgidus . I Biol Chem 2004, 279:48270-4828I.

68. DeLano WL: The PyMOL Molecular Graphics System San Carlos, CA: DeLano Scientific; 2002.

69. Thompson JD, Higgins DG, Gibson T]: CLUSTAL W: improving the sensitivity of progressive multiple sequence alignment through sequence weighting, position-specific gap penalties and weight matrix choice. Nucleic Acids Res 1994, 22:4673-4680.

70. MDL Chime [http://www.mdl.com/products/framework/chime/] 